\title{
Lane departure detection for improved road geometry estimation
}

\author{
Thomas B. Schön, Andreas Eidehall, Fredrik Gustafsson \\ Division of Automatic Control \\ Department of Electrical Engineering \\ Linköpings universitet, SE-581 83 Linköping, Sweden \\ WWW: http://Www. control.isy.liu.se \\ E-mail: schon@isy.liu.se, eidehall@isy.liu.se, \\ fredrik@isy.liu.se
}

7th December 2005

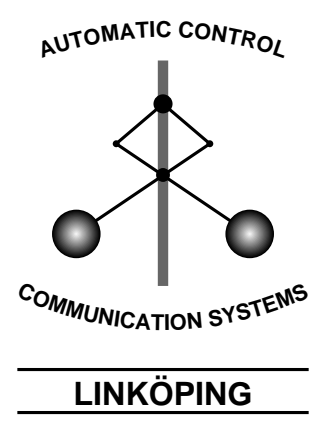

Report no.: LiTH-ISY-R-2714

Submitted to the IEEE Intelligent Vehicle Symposium 2006

Technical reports from the Control \& Communication group in Linköping are available at http://www.control.isy.liu.se/publications. 


\begin{abstract}
An essential part of future collision avoidance systems is to be able to predict road curvature. This can be based on vision data, but the lateral movement of leading vehicles can also be used to support road geometry estimation. This paper presents a method for detecting lane departures, including lane changes, of leading vehicles. This information is used to adapt the dynamic models used in the estimation algorithm in order to accommodate for the fact that a lane departure is in progress. The goal is to improve the accuracy of the road geometry estimates, which is affected by the motion of leading vehicles. The significantly improved performance is demonstrated using sensor data from authentic traffic environments.
\end{abstract}

Keywords: Automotive tracking, change detection, state estimation, Kalman filter, CUSUM-test. 


\title{
Lane departure detection for improved road geometry estimation
}

\author{
Thomas B. Schön* Andreas Eidehall ${ }^{* \dagger}$ Fredrik Gustafsson* \\ *Division of Automatic Control \\ Linköping University \\ SE-581 83 Linköping, Sweden \\ \{schon,eidehall,fredrik\}@isy.liu.se \\ ${ }^{\dagger}$ Vehicle Dynamics and Active Safety \\ Volvo Car Corporation \\ SE-405 31 Göteborg, Sweden \\ aeidehal@volvocars.com
}

\begin{abstract}
An essential part of future collision avoidance systems is to be able to predict road curvature. This can be based on vision data, but the lateral movement of leading vehicles can also be used to support road geometry estimation. This paper presents a method for detecting lane departures, including lane changes, of leading vehicles. This information is used to adapt the dynamic models used in the estimation algorithm in order to accommodate for the fact that a lane departure is in progress. The goal is to improve the accuracy of the road geometry estimates, which is affected by the motion of leading vehicles. The significantly improved performance is demonstrated using sensor data from authentic traffic environments.
\end{abstract}

Index Terms-Automotive tracking, change detection, state estimation, Kalman filter, CUSUM-test

\section{INTRODUCTION}

This paper is concerned with the problem of simultaneously estimating the position of surrounding vehicles and the road geometry. The position of the surrounding vehicles is measured using a vision system and a radar, whereas the shape of the road is measured using vision only. It has been shown that integrating the tracking of other vehicles with the tracking of the road geometry parameters can give better performance than treating these problems separately $[1,4,6,16]$. A fundamental assumption is that leading vehicles will keep following their lane, and their lateral movement can thus be used to support the otherwise difficult process of road geometry prediction. For example, when entering a curve as in Fig. 1 it can be seen that the vehicles ahead all start moving to the right and thus there is a high probability that the road is turning to the right. This information can be used to significantly improve the rather crude road geometry estimates provided by the vision system. The assumption introduced above can mathematically be represented as $\dot{y}^{i}=0$, where $y^{i}$ is the lateral position of vehicle $i$. Note that $y^{i}$ is the position in relation to the lane, not the position in global Cartesian coordinates or coordinates attached to the host vehicle. In order to efficiently handle this, a road aligned, curved coordinate system is employed. It is important to note that the assumption of zero lateral velocity of the leading vehicles does not hold when they depart from the lane. This is typically accounted for in the model by adding white noise to the equation. The amount of noise, parameterized by the covariance matrix $Q_{\text {lat }}$, that should be used is a compromise. On the one hand it needs to be small enough for the lateral movement of the tracked vehicles to in fact improve the road prediction. On the other hand, it needs to be large enough so that a lane departure of a leading vehicle is not misinterpreted as a curve entry. This exemplifies the fundamental compromise present in all recursive estimation problems, the trade-off between noise attenuation and tracking accuracy. This compromise is illustrated in Fig. 2, where the estimated road curvature, one of the road geometry parameters, using two different filters is plotted; one filter with a high value of $Q_{\text {lat }}$ and one filter with a low. For reasons of comparison, the true values for the road curvature which is obtained using the method proposed in [7] and the raw measurements from the vision system are also included. It is interesting to compare the raw vision measurements to the result from the filter. This clearly illustrates the power of a model based sensor fusion approach.

In Fig. 2, an exit phase of a curve where the curvature drops from about $1.8 \cdot 10^{-3}\left[\mathrm{~m}^{-1}\right]$ to zero can be seen. In this particular scenario there are two leading vehicles that can support our curvature estimate, see Fig. 1 . It can be seen that the filter with a low value of $Q_{\text {lat }}$ performs much better during the curve exit and this is how we would really like to tune our filter. However, at a later stage the performance of this filter deteriorates. If the recorded video is studied, see Fig. 3, it can be seen that this performance degradation coincides exactly with a lane change of one of the leading vehicles. The filter with a higher value of $Q_{\text {lat }}$ does not suffer from this problem, but on the other hand it has a time delay in the estimate during

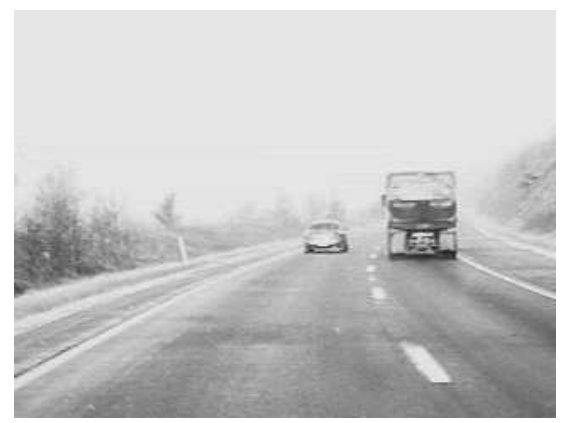

Fig. 1. When entering a curve, all vehicles start moving in the lateral direction. This information can be used to support the road geometry estimate. 


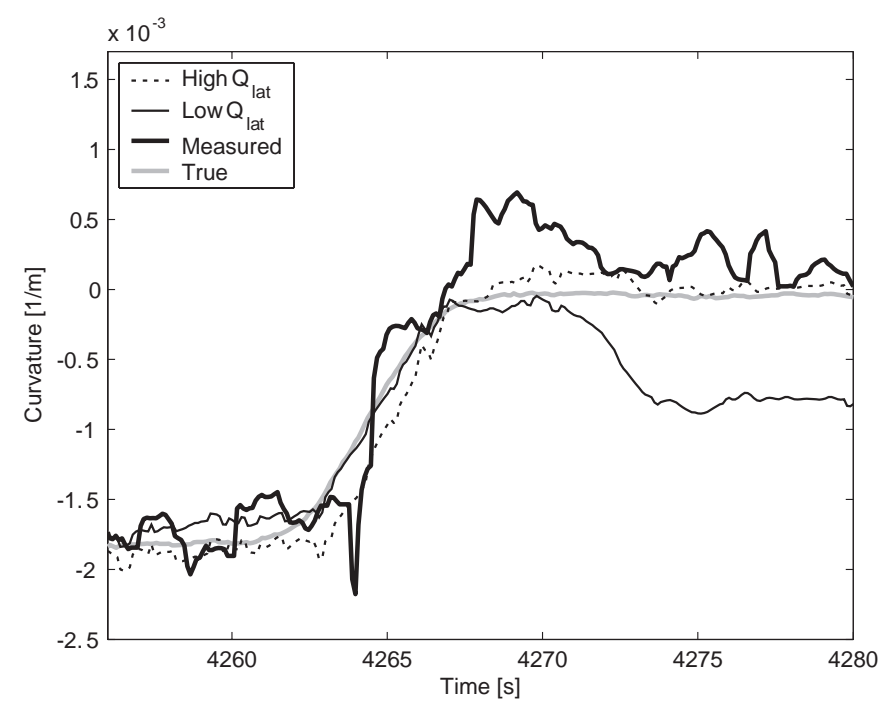

Fig. 2. Comparison of estimation performance from two filters, one with a large $Q_{\text {lat }}$ and one with a small $Q_{\text {lat }}$. The raw measurement signal from the image processing unit and the reference signal are also included. Comparing the raw vision measurement to the result from the filters clearly illustrates the power of a model based sensor fusion approach.

the curve exit.

The aim of this paper is to detect lane departures of the leading vehicles and adapt the models accordingly, in order to obtain an improved road geometry estimate. When the lane departures have been detected, the compromise discussed above can systematically be resolved. This is accomplished by using a small $Q_{\text {lat }}$ when the assumption $\dot{y}_{i}=0$ is valid and only increase $Q_{\text {lat }}$ during lane departure maneuvers.

Detection of lane departures and other model changes in automotive tracking has previously been studied, for example in [10] and [14], where Interacting Multiple Models (IMM) [2] are used. However, their purpose is to improve the position estimates of the surrounding objects, rather than the road geometry parameters. Another approach is presented in [15], where a neural network is used to detect lateral movement in a vision based system. The method we propose is different and based on the standard cumulative sum (CUSUM) algorithm [9, 12], which is augmented with a module for correcting the error caused by using the wrong model during the detection phase, before the CUSUM algorithm alarms.

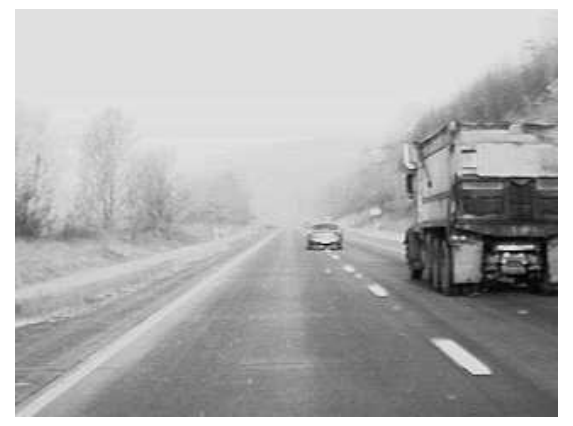

Fig. 3. A snapshot from the video just after time 4270 [s], where the lane change of the tracked vehicle commences.
The paper is structured as follows. First, the dynamic model and the estimation algorithm are briefly reviewed in Section II. This is followed by a discussion on how to detect lane departures of leading vehicles and how this information can be used to obtain better estimates. In Section IV it is discussed how the error caused by using the wrong model during the detection phase can be corrected. Finally, we provide a discussion on alternative methods in Section $\mathrm{V}$ and state our conclusion in Section VI.

\section{Estimation PRoblem}

The dynamic model is based on a curved, road-aligned coordinate system, defined in Fig. 4, where $x$ is the longitudinal position along the road and $y$ is the lateral position perpendicular to $x$. For example, this means that if $y^{i}$ is the lateral position of object $i$, then $y^{i}=0$ simply means that object $i$ is at the center of our own lane, irrespective of road shape. For the lateral dynamics, a constant position model is used, i.e., $\dot{y}^{i}=0$, and for the longitudinal dynamics a constant velocity model is used. Other states in the model are lane width $W$, host vehicle lateral position $y_{\text {off }}$, host vehicle heading angle relative to the lane $\Psi_{\text {rel }}$, road curvature parameter $c_{0}$, which is defined as the inverse road radius and finally the road clothoid parameter $c_{1}$, i.e. the curvature change rate. The vision system delivers estimates of $W, y_{\text {off }}, \Psi_{\text {rel }}$ and $c_{0}$, which are used as measurements in our estimation problem. Furthermore, the radar provides measurements of the relative position of objects resolved in the coordinate system $(\tilde{x}, \tilde{y})$, attached to the host vehicle. The dynamic model is discussed in more detail in the Appendix and the resulting estimation problem and its solution is treated in $[5,6,8]$. Tuning of the process and measurement noise will not be discussed in detail, except for the process noise of $y^{i}$. The discrete-time dynamic model describing the evolution of $y^{i}$ over time is given by

$$
y_{t+1}^{i}=y_{t}^{i}+w_{t}^{i}
$$

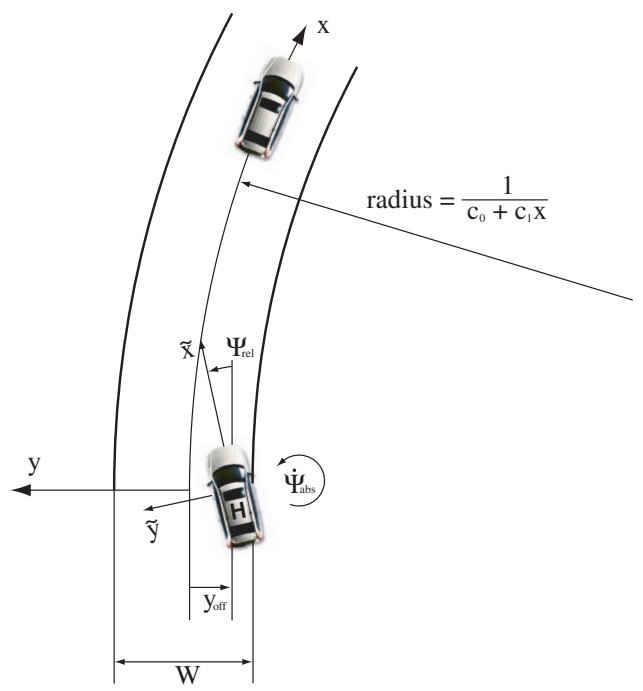

Fig. 4. The surrounding vehicles are conveniently modeled and tracked using a curved, road-aligned coordinate system $(x, y)$. 
where $w_{t}^{i}$ is zero mean white Gaussian noise, with variance $Q_{\text {lat }}$. In applying an Extended Kalman Filter (EKF), the tuning parameter $Q_{\text {lat }}$ describes to what degree it is believed that vehicles will keep driving at the same lateral position in relation to the lane.

\section{Detecting LANe Departures}

The approach employed for improving the road geometry estimates based on detecting lane departures is illustrated in Fig. 5. This is a standard approach within the area of change

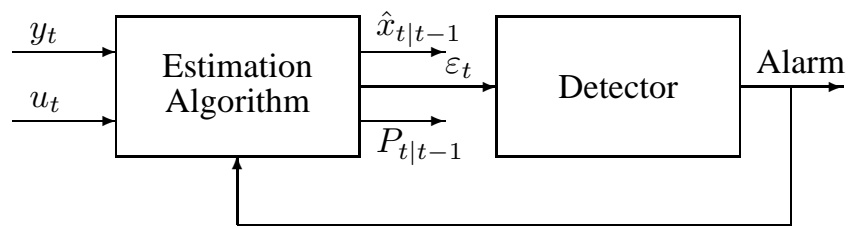

Fig. 5. The estimation algorithm delivers residuals, which are used in the detector to decide whether a change has occurred or not. If a change is detected this information is fed back for use in the estimation algorithm. Note that in this application, one detector for each tracked vehicle is needed.

detection, which is a well established research area, see e.g. $[3,9,11]$. The aim of the detector in Fig. 5 is to detect lane departures based on the information available in the residuals $\varepsilon_{t}=y_{t}-\hat{y}_{t}$ from the estimation algorithm. When a lane departure is detected this is indicated by an alarm from the detector, which is used to temporarily change model for the vehicle performing the lane departure. This implies that the estimation algorithm can provide a better estimate, simply due to the fact that a more accurate model is used. This section is concerned with devising the detection algorithm illustrated with the detection box in Fig. 5. The estimation algorithm used in the present studies is based on the extended Kalman filter $[5,6]$. The basic components of a change detection algorithm are illustrated in Fig. 6.

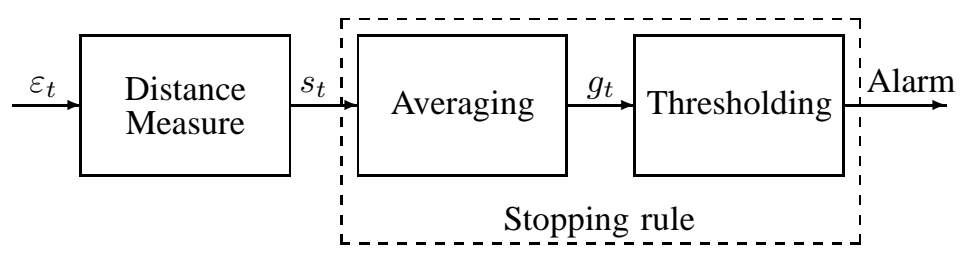

Fig. 6. The components of the detector are a distance measure, and a stopping rule, where the latter consists of an averaging and a thresholding procedure.

\section{A. Distance Measure}

The distance measure is used to assess whether a change has occurred or not. It is an important design variable, that should be chosen with the application in mind. Common standard choices are to use the residuals $s_{t}=\varepsilon_{t}$ or the squared residuals $s_{t}=\varepsilon_{t}^{2}$. However, in the present application this would provide poor detection performance. The reason is that the residuals only contain angular information. This would imply that the distance measure implicitly depend on the longitudinal distance to the leading vehicle, whereas for detecting lane departures we are only interested in lateral distances. If the longitudinal distance to the leading vehicle is small, a small change of its lateral position would lead to a large angular change. If the same change of lateral position would be observed for a vehicle further away, the angular change would be smaller. Hence, we need a distance measure that is invariant to the distance to the leading vehicle. The most natural choice in this respect is provided by lateral displacement of the leading vehicle, approximately given by

$$
s_{t}=\left|\varepsilon_{t} r_{t}\right|,
$$

where $r_{t}$ denotes the distance to the leading vehicle, available from the estimation algorithm, primarily based on the radar measurements. The reason for using $\left|\varepsilon_{t} r_{t}\right|$ and not just $\varepsilon_{t} r_{t}$ in (2) is that we want to be able to detect both left and right lateral displacements, using a one-sided test.

\section{B. Stopping Rule}

The stopping rule is used to give an alarm when an auxiliary test statistic $g_{t}$ exceeds a certain threshold. One of the most powerful tools for obtaining a good stopping rule in change detection problems is provided by the CUSUM-test, introduced by Page [12].

\section{ALGORITHM 1 (CUSUM-test):}

1) $g_{t}=g_{t-1}+s_{t}-\nu$,

2) If $g_{t}>h$ : Alarm, $g_{t}=0$ and $t_{\text {alarm }}=t$,

3) If $g_{t}<0: g_{t}=0$ and $\hat{t}_{\text {change }}=t$.

A rather detailed account of the CUSUM algorithm and its application in state estimation problems is provided in [9]. However, for the discussion to come we point out that the detection delay is the time delay between the actual event, in this case the start of a lane change manoeuvre, and the detection. In the CUSUM algorithm the detection delay is the time it takes for $g_{t}$ to reach the threshold $h$, i.e., $t_{\text {alarm }}-\hat{t}_{\text {change }}$.

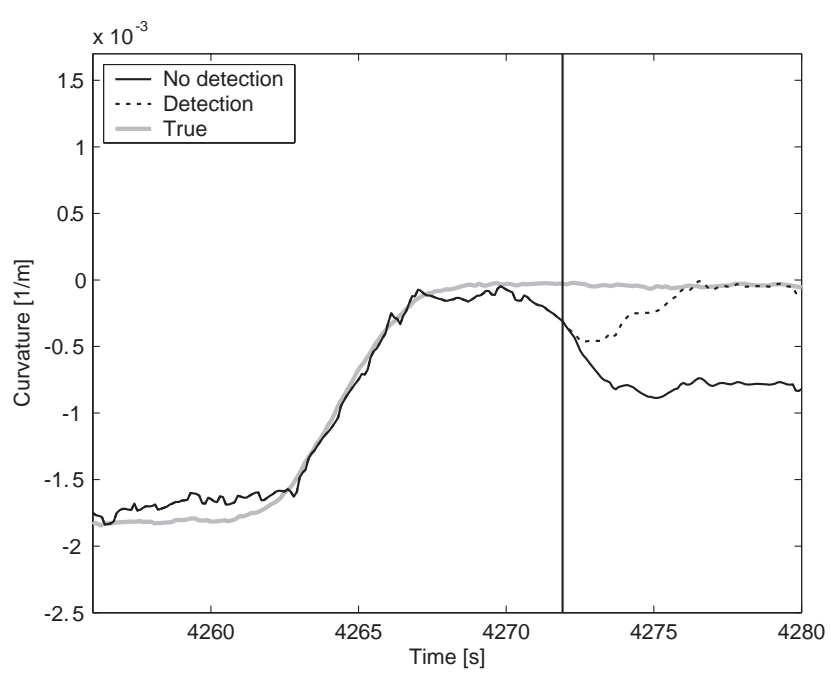

Fig. 7. Illustrating how the estimation performance is improved using lane departure detection. This is the same data using in Fig. 2, but the estimates from the filter based on change detection is also included. 
This means that when an alarm is triggered, the actual event took place a certain time ago. We will get back to this fact in Section IV, where it is used to further enhance the estimation performance.

\section{Application and Result}

When the CUSUM-test gives an alarm this is fed back to the estimation algorithm, where an increased $Q_{\text {lat }}$ is employed for the vehicle performing the lane departure. Since this model better describes the lane departure it will result in better estimates, which also is clear from Fig. 7. This lane departure model is employed during an appropriate time, corresponding to a typical lane change. After this we switch back to the original model. The idea outlined above has been tested using 35 minutes of authentic traffic data. The detection performance is detailed in Table I. For the present application a missed

TABLE I

DETECTION PERFORMANCE, BASED ON 35 MINUTES OF AUTHENTIC TRAFFIC DATA.

\begin{tabular}{l|c} 
Correct detections & 35 \\
Missed detections & 3 \\
False detections & 27
\end{tabular}

detection is much worse than false detection. A missed detection clearly degrades the estimation performance substantially, see Fig. 7, whereas a false detection merely implies a slight performance degradation, since more noise than necessary is used in the model. It is interesting, and perhaps not too surprising, to note that most of the false detections are due to sharp road turns. If these could be isolated, most of the false detections could probably be eliminated. However, since the false detections do not significantly degrade the performance this has not been investigated further.

\section{FURTHER ENHANCEMENT}

In this section, we introduce a way of correcting for the error that is caused due to the fact that the wrong model is used during the detection phase. The idea is to store old measurements $y_{t}$, input signals $u_{t}$, estimates $\hat{x}_{t \mid t}$ and covariance matrices $P_{t \mid t}$ in a memory. We propose a refiltering

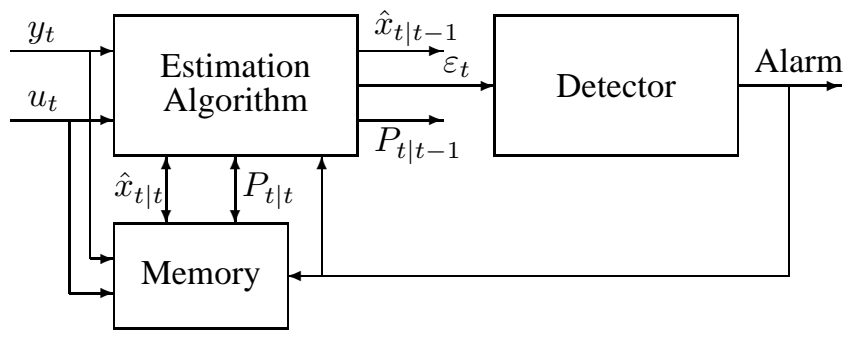

Fig. 8. The change from Fig. 5 is that a memory block has been included. The memory block stores the recent history of the measurements, input signals, estimates and their covariance.

scheme, that on detection at time $t_{\text {alarm }}$, the filter is rerun with the correct model between times $\hat{t}_{\text {change }}$ and $t_{\text {alarm }}$ in order to correct for the error that is caused by using the wrong model. The estimate at time $t_{\text {alarm }}$ is then replaced with the

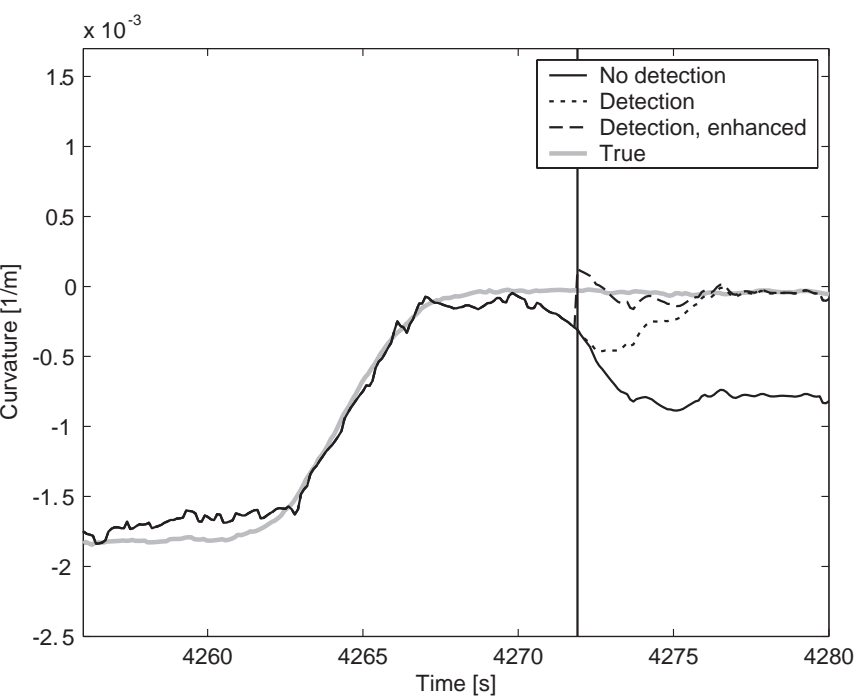

Fig. 9. The behavior of the the three approaches when the lane change is detected. The filter with no detection scheme deteriorates, the filter with detection converges when switching to the correct model, and the enhanced detection algorithm jumps to the value it would have had if it had used the correct model from the beginning of the lane change.

estimate that is obtained using the correct model. A schematic illustration of this idea is given in Fig. 8.

In our application, this means that $Q_{\text {lat }}$ is increased at time $\hat{t}_{\text {change }}$ and then kept high according to the previous section so that the total time equals the time of a typical lane change. A result of this is typically a jump in the estimate at the detection times. Two detailed examples of the behavior of the enhanced algorithm are illustrated in Fig. 9 and Fig. 10. The performance for a five minute data set is shown in Fig. 11. From this figure it is interesting to note that in the last turn, around time 4500 [s], there is a time delay in the filter which is not present in any of the other turns. This is due to the fact that there are no vehicles to support the estimate and thus the

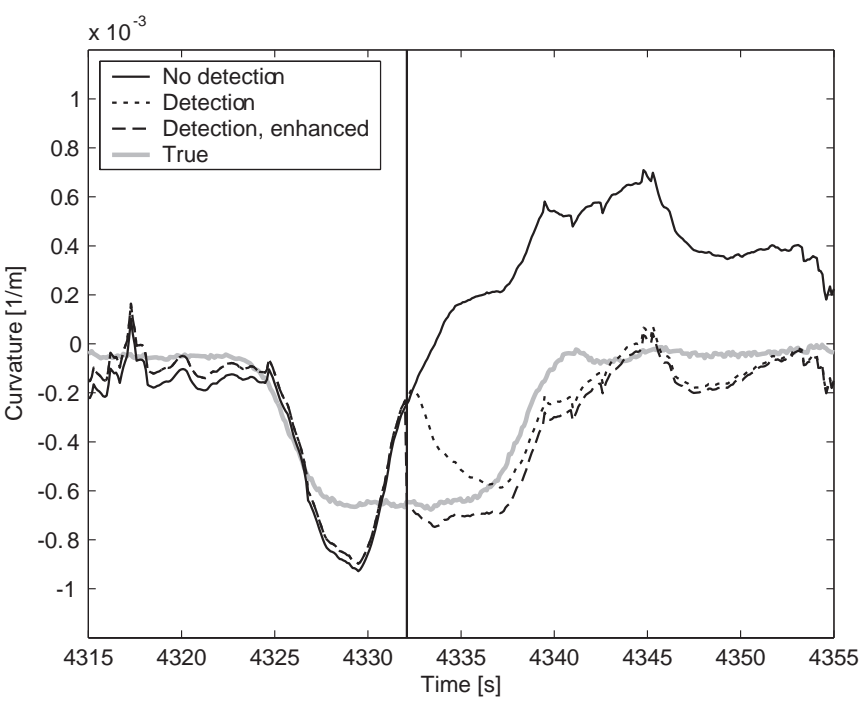

Fig. 10. Same plots as in Fig. 9 but for a different time interval. 


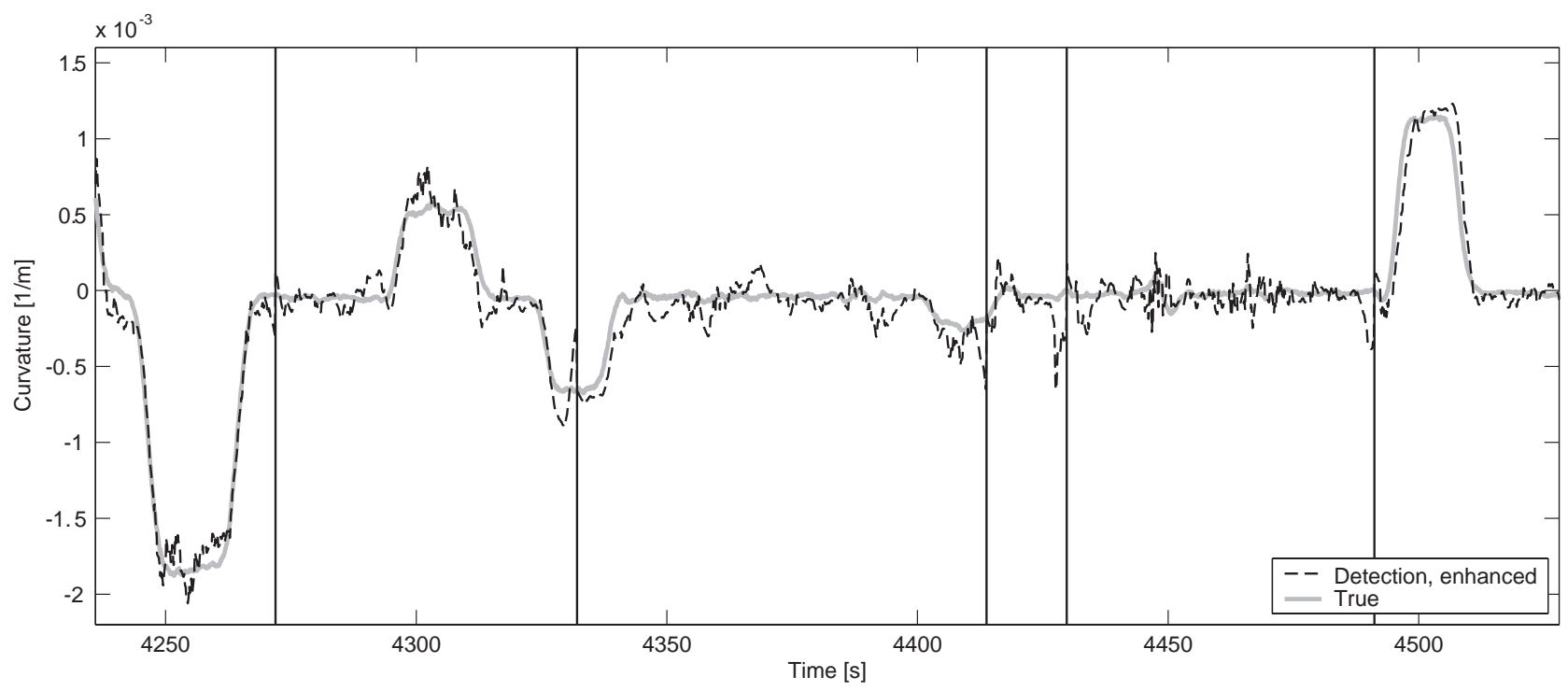

Fig. 11. This figure shows the curvature estimate for a five minute data set collected in an authentic traffic environment, compared to the true curvature value. The vertical lines indicates detection of lane changes. It is interesting to note that in the last turn, around time 4500 [s], there is a time delay in the filter which is not present in any of the other turns. This is due to the fact that there are no vehicles to support the estimate and thus the turn can only be detected robustly once we have entered it.

curve can only be detected robustly once we have entered it.

\section{Alternative methods}

The paper [14] by Weiss et al. discuss the use of a filter based on interacting multiple models (IMM) for detecting lane changes. The goal of their work is to improve the position estimates of surrounding vehicles, rather than road geometry. Of course, the same approach could be used in an integrated road geometry and object tracking model as the one proposed in this article in order to also improve road geometry estimation.

In an IMM approach, two or more models are run simultaneously and they are each given a probability, of being the "correct model", based on their residuals. The final estimate is then formed as a weighted average, using the probabilities as weights. We believe that the methods we propose here, based on the CUSUM-test, have several advantages. Firstly, a lane change is a distinct event, so either one or the other model is valid, not something in between. This means that conceptually, it is preferable to switch models completely rather than averaging two models. Secondly, the CUSUM-test provides a clear indication that something has happened, rather than a continuous change in probabilities and this indication can be used to take appropriate countermeasures. For example, this is necessary for initiating the refiltering scheme presented in Section IV.

Another idea that could be interesting to investigate is to use a two-sided test. In the proposed method, the absolute value of the residuals was used in combination with a onesided test. An alternative could be to use the signed residuals and a two-sided test, which might eliminate some of the false alarms. The reason is that an alarm could be triggered by a driver who is "wobbling" in the lane but actually not changing lanes. On the other hand, it could be argued that we would benefit from detecting any kind of lateral movement, not just lateral movement related to a lane change.

\section{CONCLUSION}

By detecting behavior that deviates from the model in a tracking system, we can rely more on the model when it in fact is accurate. In the present application, this means that the road geometry estimate, which is supported by the motion of surrounding vehicles, can be significantly improved. A CUSUM-test is used, which has the advantage of giving a distinct alarm when a change has occurred. It is also concluded that the method of correcting for the error that was caused by using the wrong model during the detection phase does give further improvements of the estimation accuracy.

\section{ACKNOWLEDGMENT}

The authors would like to thank the SEnsor Fusion for Safety systems (SEFS) program, the Intelligent Vehicle Safety Systems (IVSS) program and Volvo Car Corporation for supporting this work.

\section{APPENDIX - DYNAMIC MODEL}

In this appendix the underlying dynamic model that is used throughout the paper is discussed in more detail. The derivation is performed in continuous-time. The discrete-time dynamic is obtained using the standard sampling formula [13], under the assumption of piecewise constant input signals.

\section{System Model}

The coordinates $x$ and $y$ denote the position in the curved coordinate system, which is attached to the road according to Fig. 4. The longitudinal coordinate $x$ is relative, i.e., $x$ is the longitudinal distance between the host vehicle and 
the tracked object. The motion model for the surrounding vehicles is greatly simplified in using the curved, rather than a Cartesian coordinate system. For example, it allows us to use the equation $\dot{y}^{i}=0$, to model the assumption that the surrounding vehicles will follow their own lanes. In the longitudinal direction $\ddot{x}^{i}=-a \cos \left(\Psi_{\text {rel }}\right)$ will be used, where $a$ is the measured acceleration of the host vehicle, if available. If there are no measurements of the host vehicle's acceleration it is simply modeled as a random walk. Hence, we typically have the following motion model:

$$
\begin{aligned}
& \dot{x}^{i}=v^{i}, \\
& \dot{v}^{i}=-a \cos \left(\Psi_{\text {rel }}\right), \\
& \dot{y}^{i}=0,
\end{aligned}
$$

where $v^{i}$ is the longitudinal relative velocity of object $i$, i.e., the time derivative of $x^{i}$. It is affected by the host vehicle acceleration since it is the relative velocity that is modeled. For the road geometry parameters we first clarify that $\Psi_{\text {rel }}$ is the angle between the host vehicle and the lane, see Fig 4, whereas $\Psi_{\text {abs }}$ is the angle to some fix reference. A relationship between the two can be obtained by differentiating $\Psi_{\text {rel }}$ w.r.t. time,

$$
\begin{aligned}
& \Psi_{\text {rel }}=\Psi_{\text {abs }}+\gamma \Rightarrow \\
& \dot{\Psi}_{\text {rel }}=\dot{\Psi}_{\text {abs }}+\dot{\gamma}=\dot{\Psi}_{\text {abs }}+\frac{v}{r}=\dot{\Psi}_{\text {abs }}+c_{0} v,
\end{aligned}
$$

where $r$ is the current road radius, $v$ the velocity and $\gamma$ denotes the angle between the lane and some fix reference. $\dot{\Psi}_{a b s}$ is typically measured using a yaw rate sensor. Furthermore,

$$
\dot{y}_{\text {off }}=\sin \left(\Psi_{\text {rel }}\right) v \approx \Psi_{\text {rel }} v .
$$

Using $\dot{W}=0$ and $\dot{c}_{1}=0$ continuous-time motion equations for the road model can be written

$$
\begin{aligned}
\dot{W} & =0, \\
\dot{c}_{0} & =v c_{1}, \\
\dot{c}_{1} & =0,
\end{aligned}
$$

and for the motion of host vehicle we have

$$
\begin{aligned}
\dot{y}_{\text {off }} & =v \Psi_{\text {rel }}, \\
\dot{\Psi}_{\text {rel }} & =v c_{0}+\dot{\Psi}_{\text {abs }} .
\end{aligned}
$$

To account for uncertainties in the model we add zero mean white Gaussian noise to the corresponding discrete-time equations. The covariance matrices are $Q_{\text {road }}, Q_{\text {host }}$ and $Q_{\text {obj }}$ for the road, host and object states, respectively. Note that $Q_{\text {lat }}$, defined in Section I is the diagonal component of $Q_{\mathrm{obj}}$ corresponding to $(3 \mathrm{c})$, the lateral movement of the tracked vehicles.

\section{Measurement Model}

The measurements for the host vehicle are $\Psi_{\mathrm{rel}}^{m}, c_{0}^{m}, L^{m}$ and $R^{m}$, where the two latter are the distances to the left and right lane marking, see Fig. 4. Superscript $m$ is used to denote measured quantities. The (relative) position $\left(\tilde{x}^{m}, \tilde{y}^{m}\right)$ of the surrounding vehicles is measured using radar. Note that the radar delivers measurements resolved in the Cartesian coordinate system, which is attached to the vehicle. The resulting measurement model is,

$$
\begin{aligned}
L^{m} & =W / 2-y_{\mathrm{off}} \\
R^{m} & =-W / 2-y_{\mathrm{off}}, \\
\Psi_{\mathrm{rel}}^{m} & =\Psi_{\mathrm{rel}}, \\
c_{0}^{m} & =c_{0}, \\
\left(\begin{array}{c}
\tilde{x}^{i, m} \\
\tilde{y}^{i, m}
\end{array}\right) & =\frac{R\left(-\Psi_{\mathrm{rel}}\right)}{c_{0}}\left(\begin{array}{c}
\left(1+c_{0} y^{i}\right) \sin \left(c_{0} x^{i}\right) \\
\left(1+c_{0} y^{i}\right) \cos \left(c_{0} x^{i}\right)-1-c_{0} y_{\mathrm{off}}
\end{array}\right),
\end{aligned}
$$

where $R\left(-\Psi_{\text {rel }}\right)$ is a rotational matrix performing clockwise rotation of $\Psi_{\text {rel }}$ radians. Furthermore, zero mean Gaussian white measurement noise is added to (8). The covariance matrices are $R_{\text {host }}$ and $R_{\text {obj }}$ for the host/road and object states, respectively.

\section{REFERENCES}

[1] Automotive collision avoidance system field operational test. Technical report, National Highway Traffic Safety Administration, 2000.

[2] Y. Bar-Shalom and X.-R. Li. Estimation and Tracking: Principles, Techniques, and Software. Artech House, Norwood, MA, USA, 1993.

[3] M. Basseville and I. V. Nikiforov. Detection of abrupt changes theory and applications. Information and systems sciences series. Prentice Hall, Englewood Cliffs, NJ, USA, 1993.

[4] F. Dellaert and C. Thorpe. Robust car tracking using Kalman filtering and Bayesian templates. In Proceedings of the SPIE conference on Intelligent Transportation Systems, volume 3207, October 1997.

[5] A. Eidehall. An Automotive Lane Guidance System. Division of Automatic Control, Linköping university, Linköping, Sweden, November 2004. Licentiate Thesis No. 1122.

[6] A. Eidehall and F. Gustafsson. Combined road prediction and target tracking in collision avoidance. In Proceedings of IEEE Intelligent Vehicles Symposium, pages 619-624, Parma, Italy, June 2004.

[7] A. Eidehall and F. Gustafsson. Road g. est. In Proceedings of IEEE Intelligent Vehicles Symposium, Tokyo, Japan, June 2006. Submitted.

[8] A. Eidehall, T. B. Schön, and F. Gustafsson. The marginalized particle filter for automotive tracking applications. In Proceedings of the IEEE Intelligent Vehicle Symposium, pages 369-374, Las Vegas, USA, June 2005 .

[9] F. Gustafsson. Adaptive Filtering and Change Detection. John Wiley \& Sons, Ltd., 2000.

[10] N. Kaempchen, K. Weiss, M. Schaefer, and K. C. J. Dietmayer. IMM object tracking for high dynamic driving maneuvers. In Proceedings of IEEE Intelligent Vehicles Symposium, pages 825-830, Parma, Italy, June 2004

[11] S. K. Kay. Fundamentals of Statistical Signal Processing, Volume 1: Detection Theory. Prentice Hall Signal Processing. Prentice Hall, Upper Saddle River, New Jersey, 1998.

[12] E. S. Page. Continuous inspection schemes. Biometrika, 41(1/2):100115, June 1954.

[13] W. Rugh. Linear System Theory. Information and system sciences series. Prentice Hal, Upper Saddle River, New Jersey, 2 edition, 1996.

[14] K. Weiss, N. Kaempchen, and A. Kirchner. Multiple-model tracking for the detection of lane change maneuvers. In Proceedings of the IEEE Intelligent Vehicles Symposium, pages 937-942, Parma, Italy, June 2004.

[15] P.-S. Yen, C.-Y. Fang, and S.-W. Chen. Motion analysis of nearby vehicles on a freeway. In Proceedings of IEEE International Conference on Networking, Sensing \& Control, pages 903-908, Taipei, Taiwan, March 2004.

[16] Z. Zomotor and U. Franke. Sensor fusion for improved vision based lane recognition and object tracking with range-finders. In Proceedings of the IEEE Conference on Intelligent Transportation Systems, November 1997. 


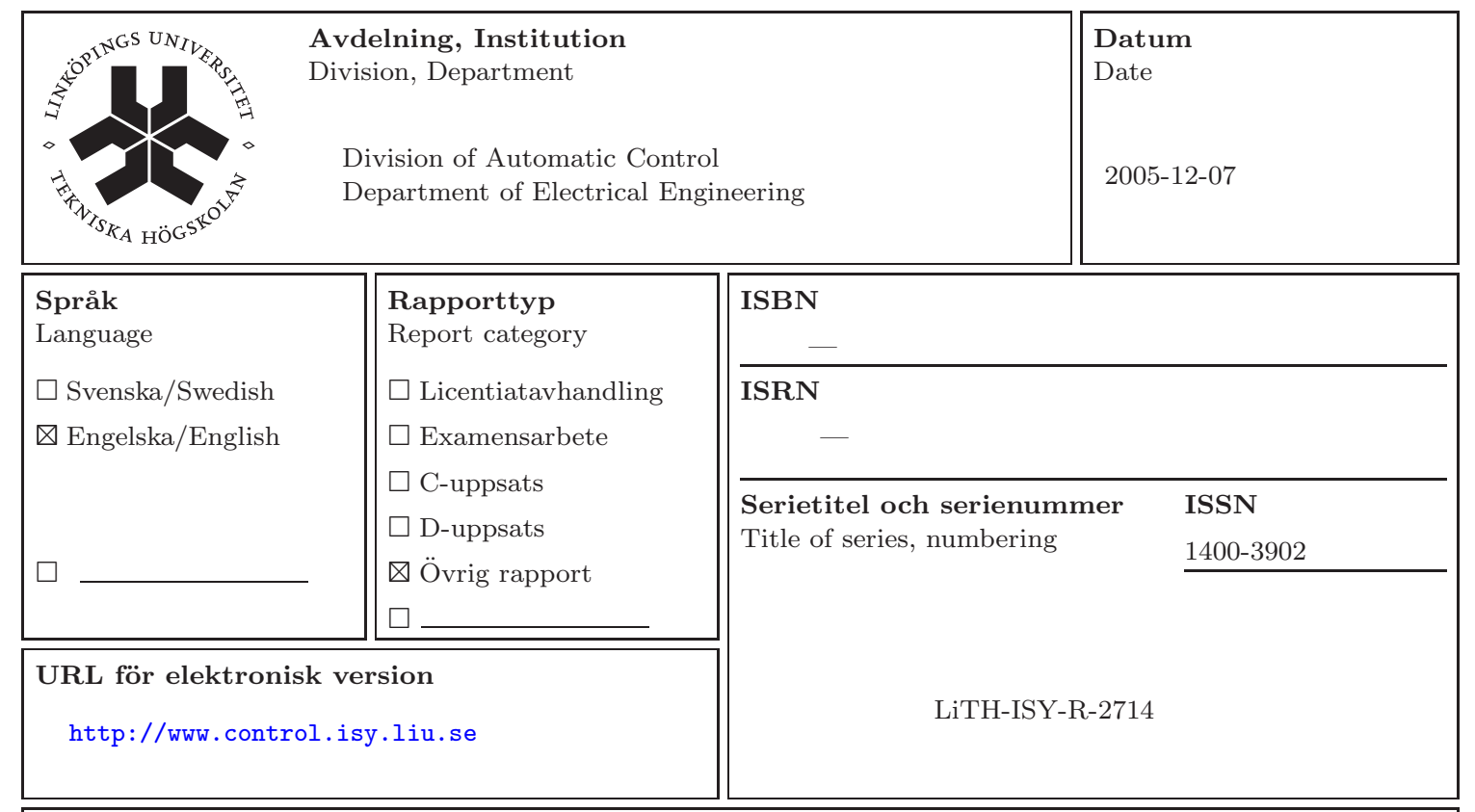

Titel Lane departure detection for improved road geometry estimation

Title

Författare Thomas B. Schön, Andreas Eidehall, Fredrik Gustafsson

Author

Sammanfattning

Abstract

An essential part of future collision avoidance systems is to be able to predict road curvature. This can be based on vision data, but the lateral movement of leading vehicles can also be used to support road geometry estimation. This paper presents a method for detecting lane departures, including lane changes, of leading vehicles. This information is used to adapt the dynamic models used in the estimation algorithm in order to accommodate for the fact that a lane departure is in progress. The goal is to improve the accuracy of the road geometry estimates, which is affected by the motion of leading vehicles. The significantly improved performance is demonstrated using sensor data from authentic traffic environments.

Nyckelord

Keywords Automotive tracking, change detection, state estimation, Kalman filter, CUSUM-test. 